\title{
Aplicação de isótopos estáveis de carbono na avaliação da taxa de crescimento do coral Porites astreoides (Lamarck, 1816) do Atol das Rocas - Atlântico Sul
}

Aline Evangelista da Silva Santos ${ }^{1 *}$ Natan Silva Pereira ${ }^{1}$ Alcides Nobrega Sial ${ }^{2}$

${ }^{1} \mathrm{NESP}$ - Núcleo de Estudos em Sedimentologia e Paleoclimatologia

Universidade do Estado da Bahia

Campus VIII

Paulo Afonso BA Brasil.

CEP 48608-240

${ }^{2}$ NEG-LABISE

Universidade Federal de Pernambuco

C. P. 7852 ,

Recife PE Brasil.

CEP 50670-000

*Autora correspondente

alineevangelistass@hotmail.com

\section{RESUMO}

Os corais são frequentemente utilizados como arquivos naturais para investigações climáticas. Esses organismos possuem a capacidade de incorporar dados geoquímicos em seus exoesqueletos, os quais podem ser utilizados como proxies para diferentes variáveis ambientais. Neste trabalho foram utilizados múltiplos perfis de isótopos estáveis de carbono $\left(\delta^{13} \mathrm{C}\right) \mathrm{em}$ diferentes seções de uma colônia de coral da espécie Porites astreoides (PTM1), coletada no Atol das Rocas - Atlântico Sul. Essa abordagem visa verificar a eficácia desse método como uma alternativa para a avaliação da taxa de crescimento de corais do grupo Scleractinia. Tal método se fundamenta na ideia que o $\delta^{13} \mathrm{C}$ de corais varia de acordo com a luminosidade (i.e. cobertura de nuvens), a qual apresenta variabilidade sazonal e deixam registrado nos corais ciclos anuais. Assim, o comprimento de cada ciclo isotópico corresponde a taxa de crescimento anual de um coral. Neste trabalho foram realizados 4 perfis isotópicos com resolução de amostragem de $0,5 \mathrm{~mm}$. Os ciclos de $\delta^{13} \mathrm{C}$ foram utilizados para avaliar a taxa de crescimento da colônia PTM-1. O comprimento dos ciclos de $\delta^{13} \mathrm{C}$ variaram de 3 a $12 \mathrm{~mm}$ ao longo dos perfis e a média geral da taxa de crescimento estimada para esta colônia foi de $6,69 \pm 2,39 \mathrm{~mm} / \mathrm{ano}$, valor próximo aos reportados para essa espécie em outras regiões, indicando a eficácia deste método ao avaliar taxa de crescimento de corais.

Palavras-chave: Geologia isotópica, corais, arquivos naturais

\section{ABSTRACT}

Corals are often used as natural archives for climate research. These organisms have the ability to incorporate geochemical data into their exoskeletons, which can be used as proxies for different environmental variables. In this work, multiple carbon stable isotope profiles $\left(\delta^{13} \mathrm{C}\right)$ were used in different sections of a Porites astreoides (PTM-1) coral colony, collected at Rocas Atoll - South Atlantic. This approach aims to verify the effectiveness of this method as an alternative for the evaluation of coral growth rate of the Scleractinia group. Such method is based on the idea that the coral $\delta^{13} \mathrm{C}$ varies according to the luminosity (i.e. cloud cover), which presents seasonal variability and leave the annual cycles recorded in the corals. Thus, the length of each isotopic cycle corresponds to the growth rate of a coral. In this work, 4 isotopic profiles with $0.5 \mathrm{~mm}$ sampling resolution were carried out. The $\delta^{13} \mathrm{C}$ cycles were used to evaluate the growth rate of the PTM-1 colony. The length of the $\delta^{13} \mathrm{C}$ cycles varied from 3 to $12 \mathrm{~mm}$ along the profiles and the overall average growth rate estimated for this colony was $6.69 \pm 2.39 \mathrm{~mm} /$ year, close to those reported for this species in other species. regions, indicating the effectiveness of this method in assessing coral growth rate.

Keywords: Isotopic Geology, corals, natural archives 


\section{INTRODUÇÃO}

Os corais são considerados excelentes arquivos naturais que podem registrar informações em alta resolução, numa escala de décadas e centenas de anos. Esses organismos conseguem incorporar informações geoquímicas (i.e. isótopos estáveis e elementos traços) que possibilitam a investigação de mudanças ambientais ocorridas em regiões tropicais (SWART; GROTTOLI, 2003). Para prover reconstruções ambientais confiáveis utilizando esqueletos de corais escleractínios é necessário compreender como os corais crescem e registram informações sobre $o$ ambiente e como os fatores biológicos e ambientais interferem no crescimento do coral (CRUZ et al. 2003; LOUGH, BARNES, 1990).

O processo de biomineralização dos corais do grupo Scleractinia ocorre por meio da deposição contínua de carbonato de cálcio $\left(\mathrm{CaCO}_{3}\right)$, precipitada por uma fina camada de tecido vivo na superfície da colônia, tal processo acontece no espaço de calcificação, onde $\mathrm{CO}_{3}{ }^{2-}$ se combina com íons de $\mathrm{Ca}^{2+}$ para formar $\mathrm{CaCO}_{3}$ (BARNES; LOUGH, 1993), levando a um crescimento que ocorre por extensão axial e espessamento do esqueleto. Rápido espessamento combinado com uma lenta extensão aumenta a densidade do esqueleto, enquanto que rápida extensão com lento espessamento reduz sua densidade (SUN et al., 2008), levando ao desenvolvimento de bandamentos de alta e baixa densidades (HUDSON et al., 1976).

A formação de bandamentos é bastante controversa, alguns autores associam bandamentos de alta densidade a temperaturas elevadas no verão, já outros relacionam bandamentos de alta densidade a temperaturas baixas, ou seja, no inverno (LOUGH; BARNES, 1990). Segundo Wood (1983) a variável ambiental com maior influência sobre os corais é a temperatura da superfície do mar (TSM), o estresse térmico influencia no metabolismo e reprodução, além de regular a saturação da aragonita. Assim, a temperatura representa um dos principais agentes ambientais que controlam o crescimento dos corais e a formação de bandamentos (LOUGH; BARNES, 2000).

$\mathrm{O}$ carbonato resultante deste processo apresenta valores empobrecidos em $\delta^{13} \mathrm{C}$ e $\delta^{18} \mathrm{O}$ em função do fracionamento cinético durante hidratação e hidroxilação do $\mathrm{CO}_{2}$ (MCCONNAUGHEY, 1989). Em corais hermatípicos, as razões de isótopos de oxigênio e carbono são utilizadas como proxies na identificação de fatores ambientais e fisiológicos (WEBER; WOODHEAD, 1970; 1972) como temperatura, salinidade e turbidez (disponibilidade de luz) da água do mar (SWART et al., 1996; SWART, 1983). Assim, os conteúdos geoquímicos dos corais são considerados proxies (i.e. evidências indiretas sobre as condições ambientais do passado, registrado de forma ordenada e sequencial num período específico) que podem ser utilizados em investigações ambientais de ecossistemas marinho tropicais (GROTTOLI, 2001; MOYER; GROTTOLI, 2011).

Segundo Grottoli (2001) é possível avaliar a taxa de crescimento de uma colônia de coral a partir de dados geoquímicos obtidos ao longo do seu eixo de crescimento. Uma vez que alguns dados geoquímicos apresentam sazonalidade anual (e.g. isótopos estáveis de $\mathrm{C}$ e $\mathrm{O}$ ), o comprimento de cada ciclo pode ser utilizado para estimar a taxa de crescimento em uma determinada seção ao longo do eixo de crescimento de um coral.

Corais do gênero Porites, Paona $e$ Montastraea são comumente utilizados em estudos paleoclimáticos por formarem grandes colônias amplamente distribuídas e apresentarem bandamentos anuais distintos (EAKIN; GROTTOLI, 2006).

Porites astreoides é comum e difundida nos trópicos, possui cálices espaçados, apresenta coloração tipicamente amarela, podendo ser amarronzada ou esverdeada. As maiores colônias da espécie podem chegar a $50 \mathrm{~cm}$ e apresentam forma maciça com protuberâncias arredondadas. São encontradas com maior frequência em áreas bem iluminadas, em topos e laterais dos recifes. Ao longo do litoral brasileiro ocorre desde o Rio Grande do Norte até o Espírito Santo, em Fernando de Noronha e no Atol das Rocas, também apresenta registros nas Bermudas, na Flórida, nas Bahamas e no Caribe (HETZEL et al., 1994).

Diante do exposto, o principal objetivo da pesquisa consistiu em testar a utilização de ciclos de isótopos estáveis de carbono como uma ferramenta para avaliar a taxa de crescimento da espécie $P$. astreoides do Atol das Rocas, Atlântico Sul equatorial. 


\section{MATERIAL E MÉTODOS}

\section{1 ÁREA DE ESTUDO}

A Reserva Biológica do Atol das Rocas constitui a primeira reserva marinha criada no Brasil (Lei $n^{\circ} 83549$ de 5 de junho de 1979) e está situada na porção oeste do Atlântico Sul, entre as coordenadas $\left(3^{\circ} 51^{\prime} \mathrm{S}, 33^{\circ} 49^{\prime} \mathrm{W}\right)$, a 266 $\mathrm{km}$ da cidade de Natal-RN (Figura 1). Rocas cresce sobre um topo aplainado de um monte submarino o qual está inserido na margem continental brasileira, especificamente no sopé continental (PALMA, 1984).

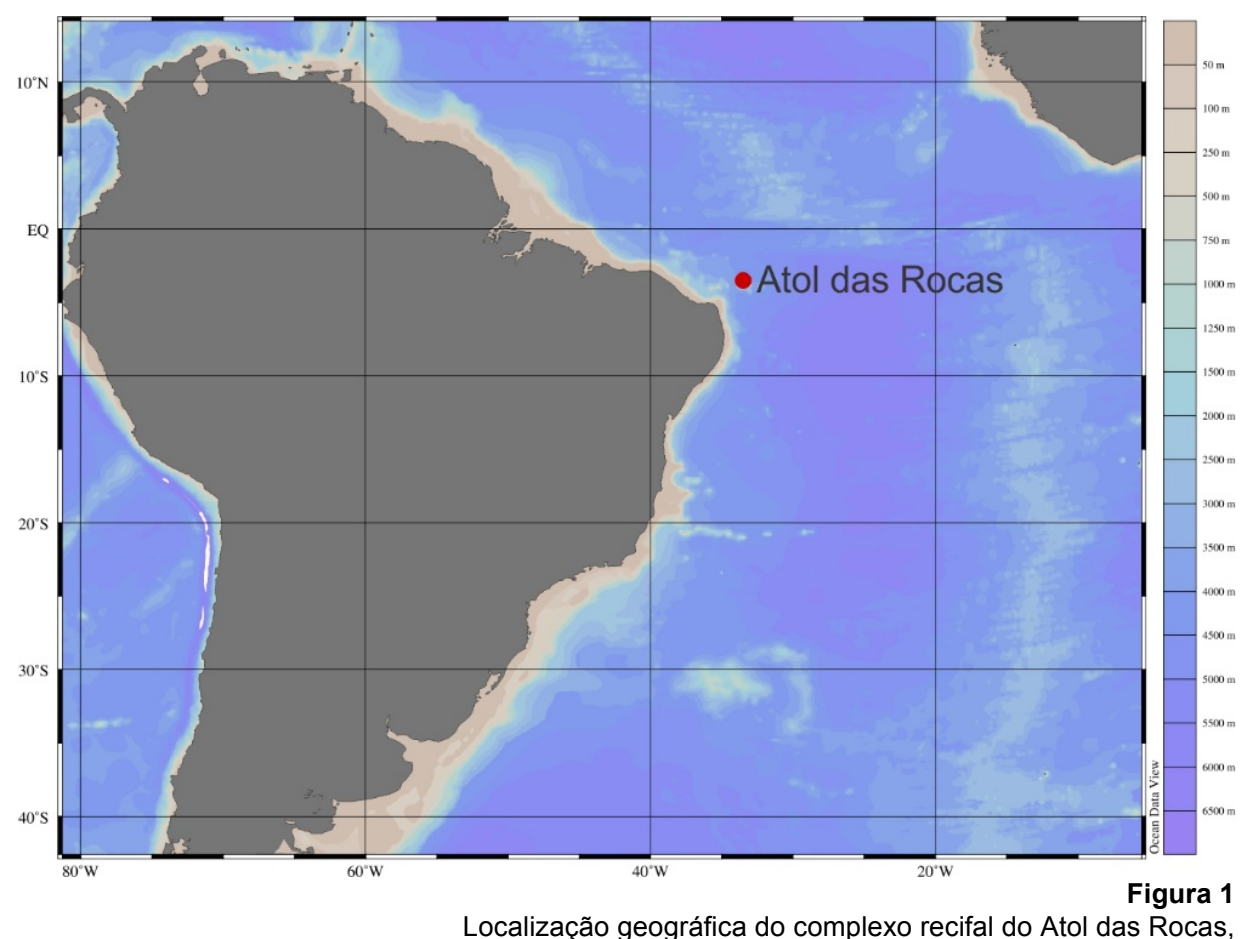

Atlântico Sul equatorial. Mapa elaborado no software Ocean Data View.

Rocas é constituído principalmente por algas coralíneas, compondo um santuário para milhares de espécies como aves e peixes, que utilizam o local para reprodução, alimentação e repouso. A superfície do recife apresenta alto índice de macroalgas, uma associação de algas coralíneas incrustantes e gastrópodes vermetídeos, espécies de corais como Siderastrea stellata (Verrill, 1868), Montastraea cavernosa (Linnaeus, 1767) e Porites $s p$. que ocorrem em áreas protegidas da energia das ondas. Em relação aos corais

\subsection{TRABALHO DE CAMPO}

A colônia de Porites astreoides foi monitorada entre outubro de 2012 e outubro de 2013, quando foi coletada (autorização ICMBio $n^{\circ}$ 28498-2) e levada ao Laboratório de Geologia e Sedimentologia (LAGES), da Universidade do Estado da Bahia (UNEB).

Para esse procedimento foram realizados mergulhos autônomos, no qual uma colônia de P. astreoides foi marcada (PTM1). Em outubro construtores de recifes é observado uma dominância de S. stellata (KIKUCHI, 2008).

O Atol das Rocas é um dos poucos locais que não sofrem interferência humana na geoquímica dos organismos ali presentes, os agentes que determinam a assinatura ambiental são o oceano e a atmosfera. Diante disso, Rocas representa um local de confiável assinatura química nos registros de corais com relação a oscilações climáticas para o Atlântico Sul (PEREIRA et al., 2017).

de 2012 a colônia foi tingida com alizarina sódica, ensacando-a em seu ambiente natural com um saco plástico, transparente de 60 litros, contendo uma concentração de $20 \mathrm{mg} / \mathrm{L}$ de alizarina, permanecendo na colônia por 24 horas. O processo foi repetido no dia posterior, perfazendo um total 48 horas de tingimento.

Para o acompanhamento da saúde da colônia e com finalidade de evitar possí- 
veis trocas por colônias não tingidas, PTM-1 foi sinalizada com dois vergalhões de ferro inseridos na periferia da colônia de modo que a mesma ficasse na parte central (Figura 2).

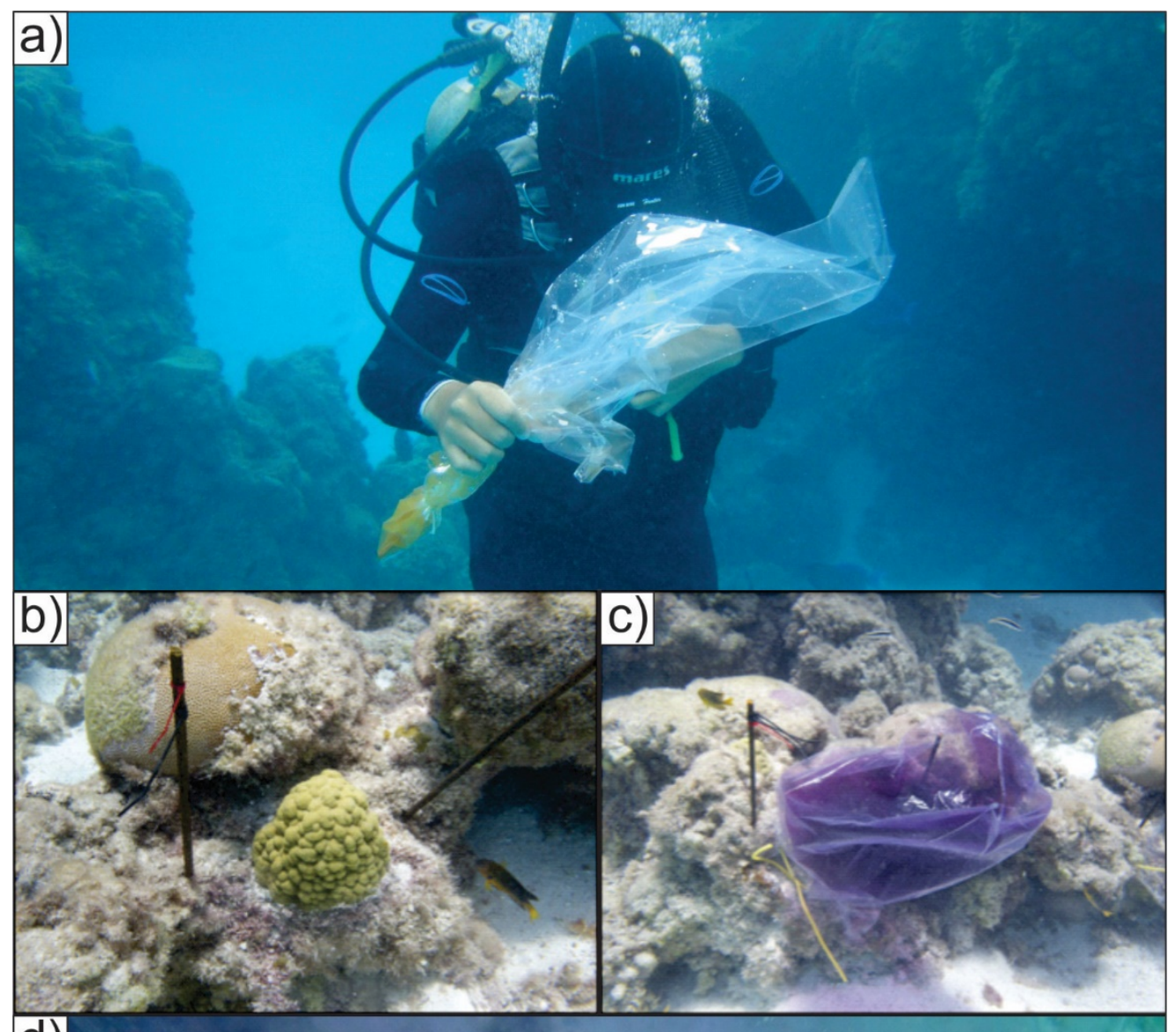

d)

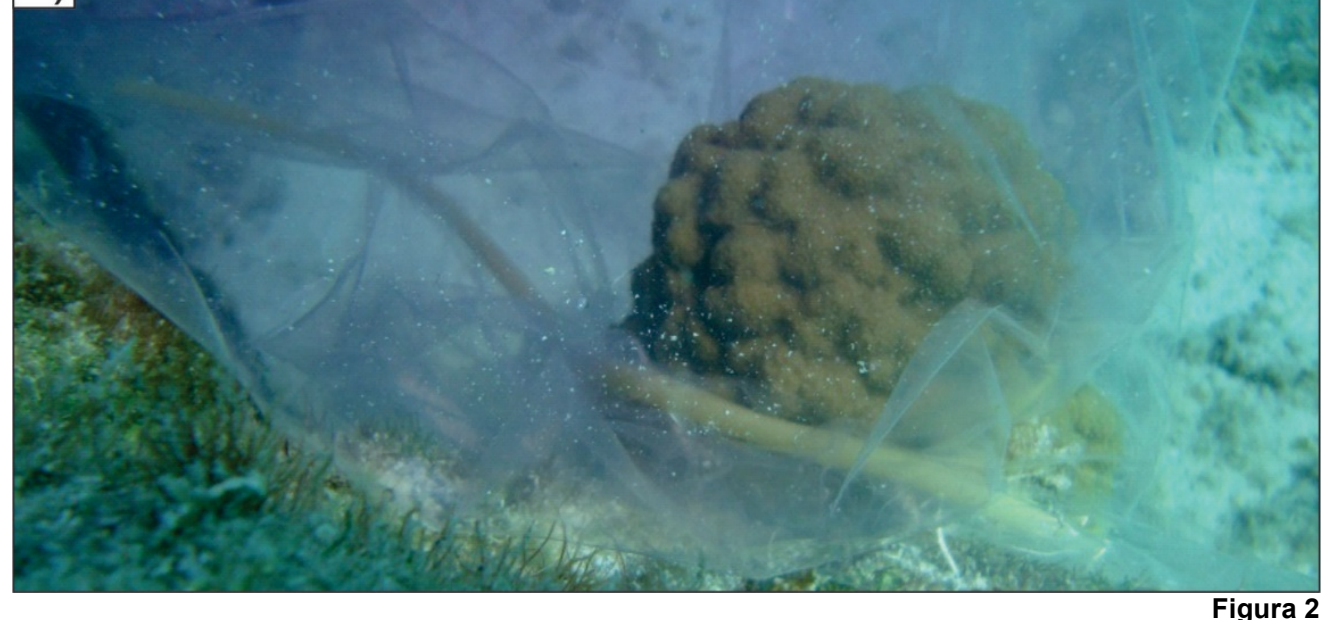

Figura 2

Procedimentos de campo mostrando a marcação da colônia com alizarina sódica em outubro de 2012. A colônia PTM-1 foi sinalizado com vergalhões e coleta após 1 ano de monitoramento. Adaptado de Pereira, 2015.

\subsection{ANÁLISE EM LABORATÓRIO}

Lâminas de $5 \mathrm{~mm}$ de espessura foram obtidas através de cortes longitudinais paralelos ao eixo de crescimento máximo de cada colônia. As lâminas que apresentaram as estruturas dos coralitos crescendo paralelamente ao corte foram selecionadas para serem radiografadas e amostradas para análises geoquímicas.

Uma microfuradeira de dentista foi utilizada para retirar amostras de coral (aragonita), com massa variando de 4 a $20 \mathrm{mg}$, ao longo de 5 seções paralelas ao eixo de 
crescimento principal da lâmina (Figura 3). A resolução de amostragem foi de $0,5 \mathrm{~mm}$. Essas amostras foram encaminhadas para análise de isótopos estáveis de $\mathrm{C}$ e $\mathrm{O}$ no Laboratório de Isótopos Estáveis da Universidade Federal de Pernambuco.

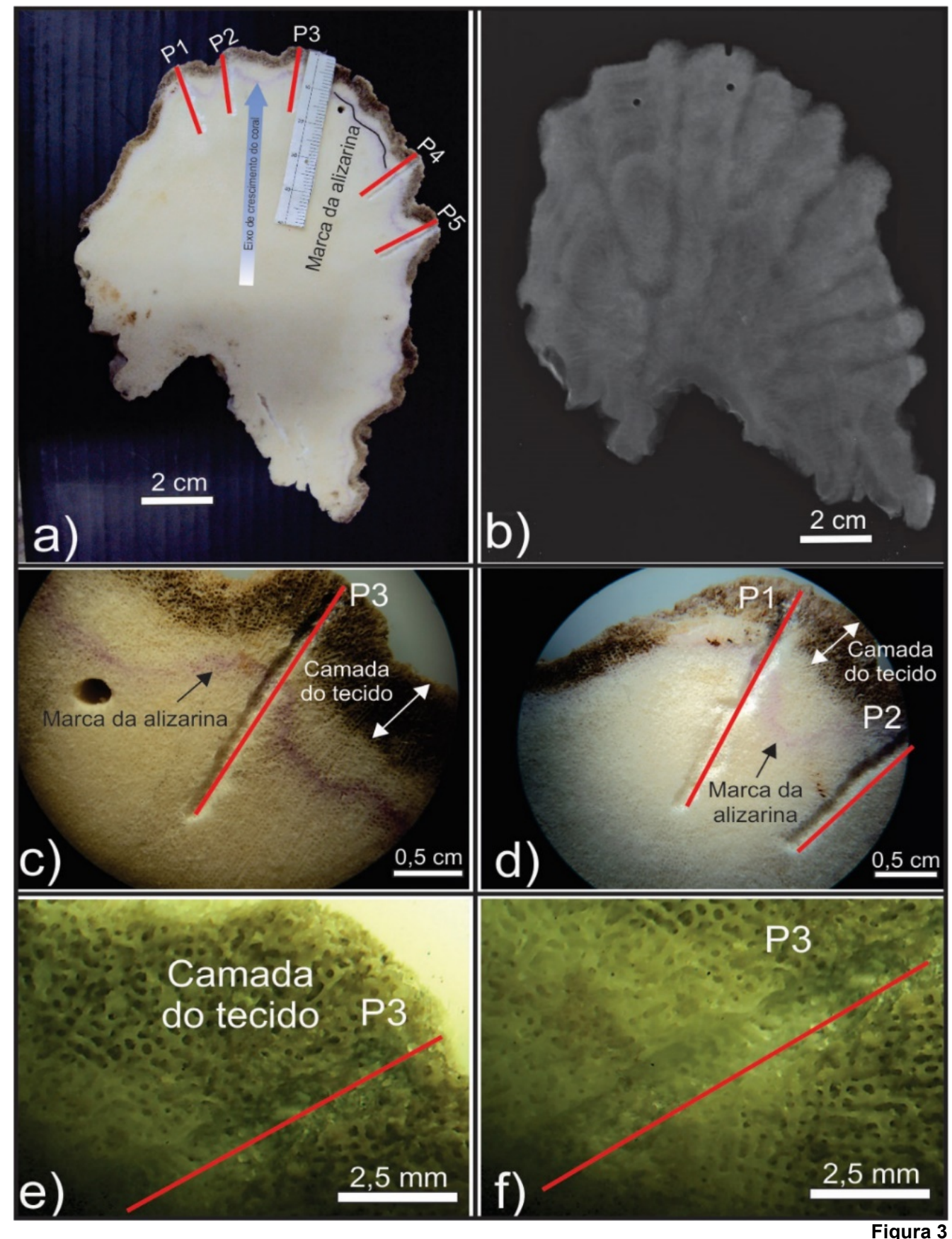

Esquema de amostragem ao longo de uma lamina de coral da espécie $P$. astreoides coletada no Atol das Rocas em Outubro de 2013: (a) amostra indicando os pontos de coletas, (b) Raio X da amostra, (c- d) camada do tecido e marca da alizarina nos perfis, (e- f) camada do tecido e corte ao longo do perfil visualizado em microscópio.

\subsection{ANÁLISES DE $\delta^{13} \mathrm{C}$}

As análises de $\delta^{13} \mathrm{C}$ foram determinadas utilizando espectrômetro de massa Delta $\mathrm{V}$ Advantage com GasBench II acoplado do Laboratório de Isótopos Estáveis (LABISE) da Universidade Federal de Pernambuco (UFPE). Para isso for necessários cerca de $0,6-0,7 \mathrm{mg}$ de carbonato que foram transferidos para frascos de vidro de $10 \mathrm{ml}$ e selados com tampas com septos de borracha.

Em cada rodada de análise foram processados 70 frascos usando uma unidade de multifluxo, dos quais 16 frascos continham 4 
padrões de referência com valor isotópico conhecido, sendo 2 internacionais (NBS-18 e NBS-19) e dois padrões internos (REI e BSC) para calibração.

Inicialmente, os frascos passaram por um flush de Hélio (He), que consiste em um jato de gás $\mathrm{He}$ por 240 segundos para remoção de gás atmosférico e água, especialmente $\mathrm{CO}_{2} \mathrm{e}$ $\mathrm{H}_{2} \mathrm{O}$. O gás foi inserido por uma agulha introduzida através do septo de borracha na qual a mistura de gases foi continuamente removida por um canal de escape aberto na

\section{RESULTADOS E DISCUSSÃO}

\subsection{ISÓTOPOS ESTÁVEIS DE CARBONO $\left(\delta^{13} \mathrm{C}\right)$}

Os valores isotópicos obtidos na análise geral da amostra PTM-1 para o $\delta^{13} \mathrm{C}$ apresentam uma média de $-0,91 \pm 0,38 \%$ VPDB, mínimo de -2,09\% VPDB, máximo de $0,07 \%$ VPDB com variação de $2,16 \%$ VPDB. Nas análises, o perfil 1 apresentou uma média de $-0,98 \pm 0,25 \%$ o VPDB, mínimo de $-1,43 \%$ 。 VPDB, máximo de - $0,25 \%$ VPDB, variação de 1,18\% VPDB. O perfil 2 apresenta uma média de $-0,84 \pm 0,36 \%$ VPDB, com mínimo de 2,09\% VPDB, máximo de -0,22\% VPDB, variação de 1,86\% VPDB. O perfil 3 apresenta uma média de $-0,64 \pm 0,32 \%$ VPDB, mínimo de $-1,25 \%$ o VPDB, máximo de 0,07\%o VPDB, variação de 1,32\% VPDB. O perfil 4 apresenta uma média de $-1,26 \pm 0,29 \%$ VPDB, mínimo de -2,07\% VPDB, máximo de -0,78\% VPDB, variação de 1,29\% VPDB (Tabela 1).

Os principais fatores que governam a assi- agulha introduzida.

Após o flush de $\mathrm{He}, 90 \mathrm{mg}$ (equivalente a $\sim 50 \mu \mathrm{l})$ de ácido fosfórico $\left(\mathrm{H}_{3} \mathrm{PO}_{4}\right)$ foram adicionados automaticamente em cada amostra para liberar o $\mathrm{CO}_{2}$ do carbonato, através de uma agulha de aço movida por um braço robótico Comb Pal. Depois da adição de $\mathrm{H}_{3} \mathrm{PO}_{4}$, as amostras reagiram por 90 minutos antes de serem analisadas. A mistura de He$\mathrm{CO}_{2}$ de cada amostra foi então transferida em um processo automático e mensurados no DeltaV advantage.

natura isotópica do carbono ainda estão em debate. Grottoli (2001) sugeriu que os valores de $\delta^{13} \mathrm{C}$ estão associados a atividade fisiológica, influenciando principalmente na alimentação do coral. Weber e Woodhead (1972) propuseram que a variação isotópica do carbono está diretamente ligada a atividade fotossintética das zooxantelas. McConnaughey (1989), afirma que durante o processo fotossintético as zooxantelas absorvem o ${ }^{12} \mathrm{C}$, resultando em elevação de $\delta{ }^{13} \mathrm{C}$ na aragonita. Já Erez (1978) propôs que a fotossíntese ocasiona a diminuição de $\delta{ }^{13} \mathrm{C}$ nos corais. Os resultados obtidos na pesquisa de Pereira et al. (2017) indicam que durante os períodos de baixa irradiação solar, a incorporação de delta $\delta{ }^{13} \mathrm{C}$ é mais baixa. Também foram observados valores de $\delta{ }^{13} \mathrm{C}$ mais altos em setembro e mais baixos em abril no Atol das Rocas.

Tabela 1 - Comparação entre média, mínimo, máximo e variação das análises isotópicas ao longo dos 4 perfis, da colônia PTM1 para o $\delta^{13} \mathrm{C}$.

\begin{tabular}{ccccc}
\hline \multicolumn{5}{c}{$\boldsymbol{\delta}^{\mathbf{1 3}} \mathbf{C}_{(\% \text { VPDB })}$} \\
\hline Perfil & P1 & P2 & P3 & P4 \\
\hline Média & $-0,98 \pm 0,25$ & $-0,84 \pm 0,36$ & $-0,64 \pm 0,32$ & $-1,26 \pm 029$ \\
Mínimo & $-1,43$ & $-2,09$ & $-1,25$ & $-2,07$ \\
Máximo & $-0,25$ & $-0,22$ & 0,07 & $-0,78$ \\
Variação & 1,18 & 1,86 & 1,32 & 1,29 \\
\hline
\end{tabular}

\subsection{CICLOS ISOTÓPICOS E AVALIAÇÃO DA TAXA DECRESCIMENTO 3.2.1 ISÓTOPOS DE CARBONO}

Com a finalidade de definir possíveis ciclos anuais ao longo dos perfis isotópicos, foram descritas as quantidades de ciclos presentes em cada perfil, sendo o primeiro ciclo comparado com o crescimento monitorado de um ano, revelado pela marca de alizarina. Os demais ciclos, quando bem definidos, foram associados a taxa de crescimento anual do coral (Figura 4).

Assim, por meio da análise dos perfis de $\delta^{13} \mathrm{C}$ foi possível observar no perfil 1 dois ciclos isotópicos, tendo o primeiro ciclo uma 
extensão correspondente a marca da alizarina, correspondendo a um crescimento de $7,5 \mathrm{~mm} / \mathrm{ano}$, e um segundo ciclo, pouco definido, com cerca de $13 \mathrm{~mm}$. O perfil 2 apresenta dois ciclos isotópicos, com o primeiro ciclo correspondendo a $7 \mathrm{~mm}$, e um segundo ciclo com cerca de $7 \mathrm{~mm}$. O perfil 3 também apresentou dois ciclos isotópicos, tendo o primeiro ciclo $7 \mathrm{~mm}$ o segundo $5 \mathrm{~mm}$ de extensão. $\mathrm{O}$ perfil 4 apresenta 4 ciclos isotópicos, dentre os quais, dois estão dentro da extensão correspondente a um ano de crescimento (marca de alizarina), portanto, não representam ciclos anuais.
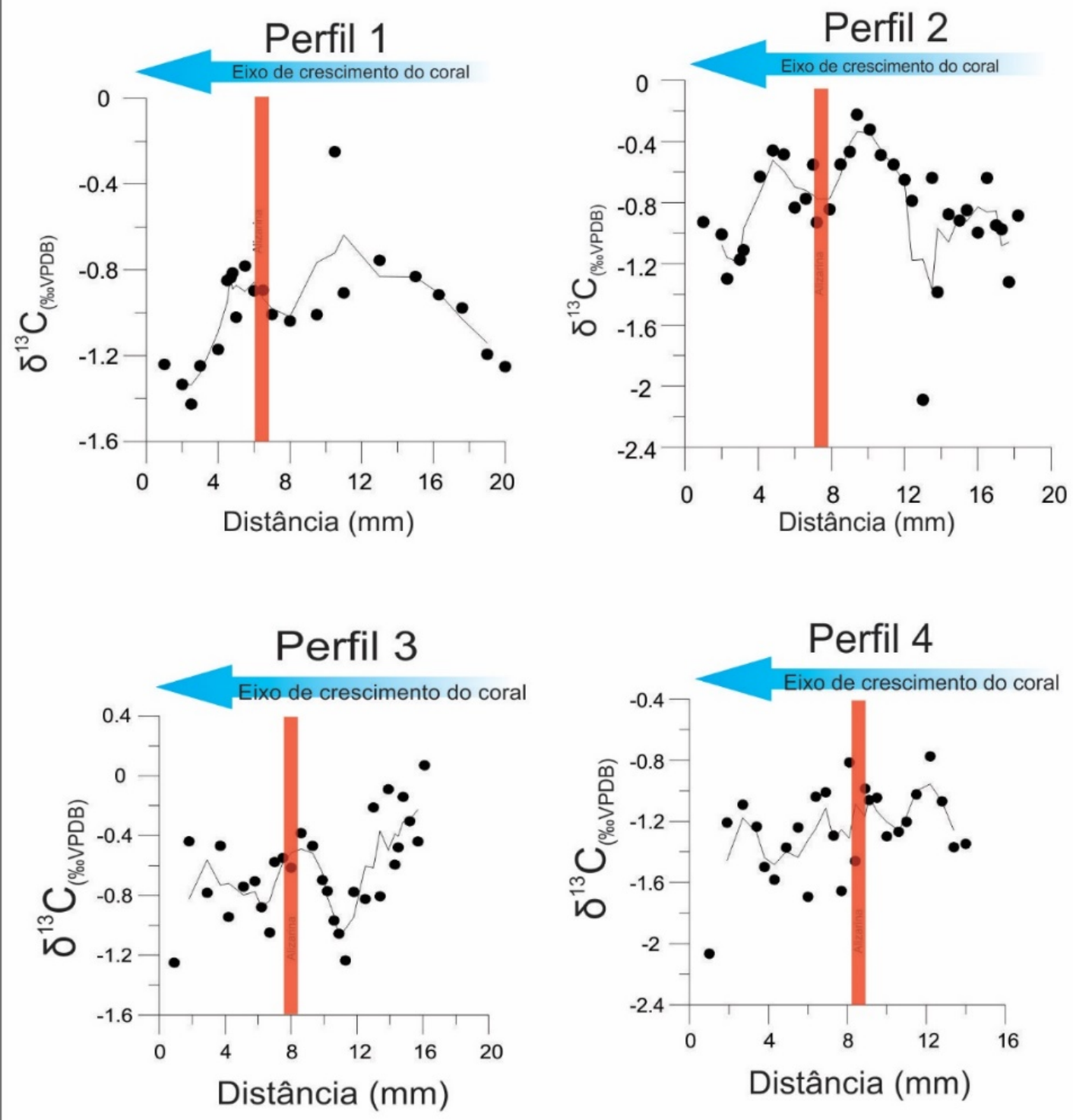

Figura 4

Ciclos isotópicos da amostra para o $\delta^{13} \mathrm{C}$ ao longo dos quatros perfis. A linha vermelha nos perfis representa a marca da alizarina.

Os ciclos isotópicos para o $\quad \delta^{13} \mathrm{C}$ identificados ao longo dos quatros perfis apresentaram uma diferença com relação aos seus valores de extensões que variaram de 3 a
$12 \mathrm{~mm}$ e a média geral da taxa de crescimento de $6,69 \pm 2,39 \mathrm{~mm} /$ ano. Os perfis de 1 a 4 apresentaram médias de 9,75, 7,6 e $4 \mathrm{~mm}$, respectivamente (Tabela 2 ). 
Tabela 2 - Valores das extensões $(\mathrm{mm})$ dos ciclos isotópicos para o $\delta^{13} \mathrm{C}$ identificados ao longo dos 4 perfis e média geral da taxa de crescimento estimada.

\begin{tabular}{llllll}
\hline Ciclos & P1 & P2 & P3 & P4 & \\
\hline 1 & 7,5 & 7 & 7 & 5 & \\
2 & 12 & 7 & 5 & 3 & 3 \\
3 & & & & 5 & \\
4 & & & & 4 & $\mathbf{6 , 6 9} \pm \mathbf{2 , 3 9} \mathbf{m m} / \mathbf{a n o}$ \\
\hline Média & 9,75 & 7 & 6 & & \\
\hline
\end{tabular}

A estimativa obtida para a taxa de crescimento de $P$. astreoides está dentro da média para o gênero em outras localidades, como P. lutea (MILNE EDWARDS; HAIME, 1851) na ilha Riau com crescimento variando de 8 a $12 \mathrm{~mm} /$ ano (SUPRIHARYONO, 2004), e P. lobata (DANA, 1846) nas Filipinas com crescimento de 3 a $13 \mathrm{~mm} / \mathrm{ano}$ (PÄTZOLD,

\section{CONSIDERAÇÕES FINAIS}

As séries temporais de $\delta^{13} \mathrm{C}$ obtidas ao longo de diferentes seções de uma colônia de P. astreoides do Atol das Rocas foram dominadas por variações de curto prazo, as quais são governadas pela variabilidade anual de cobertura de nuvens na região. $\mathrm{O}$ comprimento dos ciclos do $\delta^{13} \mathrm{C}$ revelaram uma taxa média de crescimento de 6,69 $\pm 2,39$

\section{AGRADECIMENTOS}

Agradecemos a Chefe da Reserva Biológica do Atol das Rocas, Maurizélia de Brito Silva e a equipe de campo: Tiago Albuquerque, Miguel Miranda, Mirella B.

\section{REFERÊNCIAS}

BARNES, D. J.; LOUGH, J. M. On the nature and causes of density banding in massive coral skeletons. Journal of Experimental Marine Biology and Ecology, 167(1), 91-108. 1993.

CRUZ-PIÑÓN, G.; CARRICART-GANIVET, J.P.; ESPINOZA-AVALOS, J. Monthly skeletal extension rates of the hermatypic corals Montastraea annularis and Montastraea faveolata: biological and environmental controls. Marine Biology, 143(3), 491-500. 2003.

EAKIN, C. M.; GROTTOLI, A. Paleo-climate changes and corals. Earth-Science Reviews, 81, 67-91, 2006.

EREZ, J. Vital effect on stable-isotope composition seen in foraminifera and coral skeletons. Nature, 273(5659), 199-202, 1978.

GROTTOLI, A. G. Past climate from corals. In: STEELE，J.; THORPE，K.; TUREKIAN，K.
1984). A média de crescimento está de acordo com uma pesquisa realizada por Lough e Barnes (2000), segundo os quais a taxa de crescimento do gênero Porites varia de 3,4 $12,9 \mathrm{~mm} /$ ano. Também corrobora com a pesquisa de Pereira et al. (2017), no qual obteve valor médio de $8,62 \pm 1,82 \mathrm{~mm} /$ ano para a espécies $P$. astreoides em Rocas.

mm/ano para a colônia PTM-1.

Essa pesquisa contribui para a expansão do banco de dados geoquímicos com base em corais na região do Atlântico Sul tropical, bem como, amplia a discussão sobre o potencial de diferentes espécies de corais como arquivos naturais promissores para a reconstruções peleoclimáticas nessa região.

Costa e Eduardo Macêdo pela assistência nos trabalhos de campo. Esse trabalho recebeu suporte financeiro da FAPESB (JCB0045/2016).

(Org.). Encyclopedia of ocean sciences. London: Academic Press, 2098-2107, 2001.

Hetzel, B.; CASTRO, C. B. Corais do Sul da Bahia. 1. ed. Rio de Janeiro: Nova Fronteira, 199 pp.

HUDSON, J. H.; SHINN, E,. A.; HALLEY, R. B.; LIDZ, B. Sclerochronology: A tool for interpretating past environments. Geology, 4 (6), 361-364, 1976.

KIKUCHI, R. K. P. Atol das Rocas, Litoral do Nordeste do Brasil: único atol do Atlântico Sul Equatorial Ocidental. Sítios geológicos e paleontológicos do Brasil, 8, 379-391, 2008.

LOUGH, J. M.; BARNES, D. J. Environmental controls on growth of the massive coral Porites. Journal of Experimental Marine Biology and Ecology, 245(2), p. 225- 243, 2000.

LOUGH, J. M.; BARNES, D. J. Intra-annual timing of density band formation of Porites coral from 
the central Great Barrier Reef. Journal of Experimental Marine Biology and Ecology, v. 135(1), 35-57, 1990.

MCCONNAUGHEY, T. $13 \mathrm{C}$ and $18 \mathrm{O}$ isotopic disequilibrium in biological carbonates: I. Patterns. Geochimica et Cosmochimica Acta, 53(1), 151-162, 1989.

MOYER, R. P.; GROTTOLI, A. G. Coral skeletal carbon isotopes ( $\delta 13 \mathrm{C}$ and $\Delta 14 \mathrm{C}$ ) record the delivery of terrestrial carbon to the coastal waters of Puerto Rico. Coral Reefs, 30(3), 791, 2011.

PALMA, J. J. C. Fisiografia da área oceânica. In: SCHOBBENHARES, C. (Org). Geologia do Brasil. Brasília: Ministério das Minas e Energia. Departamento Nacional de Produção Mineral, p. 501. 1984.

PÄTZOLD, J. Growth rhythms recorded in stable isotopes and density bands in the reef coral Porites lobata (Cebu, Philippines). Coral Reefs, 3(2), 87-90, 1984.

PEREIRA, N. S.; SIAL, A. N.; FREI, K.; ULlMANN, C. V.; KILBOURNE, K. H. The potential of the coral species Porites astreoides as a paleoclimate archive for the Tropical South Atlantic Ocean. Journal of South American Earth Sciences, 77, 279-285, 2017.

SUN, D,; SU, R.; MCCONNAUGHEY, T. A.; BLOEMENDAL, J. Variability of skeletal growth and $\delta 13 \mathrm{C}$ in massive corals from the
South China Sea: Effects of photosynthesis, respiration and human activities. Chemical Geology, 255(3-4), 414-425, 2008.

SUPRIHARYONO, S. Effects Of Sand Mining On Coral Reefs In Riau Islands. Journal of Coastal Development VII (2). pp. 89-100. ISSN ISSN: 1410-5217. 2004.

SWART, P. Carbon and oxygen isotope fractionation in scleractinian corals: a review. Earth-Science Reviews, v. 19, n. 1, p. 51-80. 1983.

SWART, P. K.; GROTTOLI, A. Proxy indicators of climate in coral skeletons: a perspective. Coral Reefs 22(4), 313-315, 2003

SWART, P. K.; LEDER, J. J.; SZMANT, R. E.; DODGE, R. E. The origin of variations in the isotopic record of scleractinian corals: II. Carbon. Geochimica et Cosmochimica Acta, 60(15), 2871-2885, 1996.

WEBER, J. N.; WOODHEAD, P. M. J. Temperature dependence of oxygen-18 concentration in reef coral carbonates. Journal of Geophysical Research, v. 77, n. 3, p. 463473, 20 jan. 1972.

WEBER, J.; WOODHEAD, P. Carbon and oxygen isotope fractionation in the skeletal carbonate of reef-building corals. Chemical Geology, v. 6, p. 93-117, 1970.

WOOD, E. M. Corals of the world. 1. ed. Neptune City: T. F. H. Publications, 1983. 